\title{
Conversion of Australian Food Composition Data from AUSNUT1999 to 2007 in the Clinical Trial Context
}

\author{
Elizabeth P. Neale ${ }^{1}$, Yasmine C. Probst ${ }^{1}$, Rebecca Thorne ${ }^{1}$, Qingsheng Zhang ${ }^{1}$, Jane O’Shea ${ }^{1}$, Marijka J. \\ Batterham $^{2}$ and Linda C. Tapsell ${ }^{1}$ \\ 1. Smart Foods Centre, University of Wollongong, New South Wales 2522, Australia \\ 2. Statistical Consulting Service, University of Wollongong, New South Wales 2522, Australia
}

Received: November 23, 2011 / Published: February 20, 2012.

\begin{abstract}
An Australian food composition database, AUSNUT1999, does not include long chain omega-3 polyunsaturated fatty acid (LC omega-3 PUFA) data. Measurement of the fatty acid content of diets initially analysed using AUSNUT1999 requires conversion to AUSNUT2007, an updated database inclusive of LC omega-3 PUFA. The aim of this study was to convert clinical trial dietary data from AUSNUT1999 to AUSNUT2007 and measure LC omega-3 PUFA intake. Clinical trial diet history (DH) data was converted from AUSNUT1999 to 2007 using a staged approach. Macronutrient intake from AUSNUT1999 and 2007 were calculated and compared via paired $t$-tests and Wilcoxon Signed Ranks tests. Mean dietary LC omega-3 PUFA intake and the percentage contribution of food groups to total LC omega-3 PUFA were then calculated. DHs were collected at baseline $(\mathrm{n}=118)$, three months $(n=86)$, and 12 months $(n=64)$. The accuracy of the conversion process improved with time, with no significant difference between most macronutrients at 12 months. Mean LC n-3 PUFA intake was $441.87 \mathrm{mg}$ at baseline, $521.07 \mathrm{mg}$ at $3 \mathrm{months}$, and $442.40 \mathrm{mg}$ at 12 months, and was predominantly provided by fish and seafood, followed by meat products. This study allowed for the measurement of LC omega-3 intake, which was previously impossible using the AUSNUT1999 database.
\end{abstract}

Key words: AUSNUT1999, AUSNUT2007, long chain omega-3 polyunsaturated fatty acids, database conversion.

\section{Introduction}

Consumption of long chain omega-3 polyunsaturated fatty acids (LC n-3 PUFA) has been associated with a number of health benefits, including decreased risk of heart failure and stroke [1-5], and thus is of interest from a public health perspective. However, measurement of dietary LC n-3 PUFA in the Australian context is problematic. The Australian Food and Nutrient Database (AUSNUT) 1999 is an Australian food composition survey database developed by Food Standards Australia and New Zealand (FSANZ) [6]. AUSNUT1999 is based on foods consumed in the 1995 National Nutrition Survey (NNS) [6]. AUSNUT1999 has been utilised in

Corresponding author: Elizabeth P. Neale, Ph.D. candidate, research fields: nutrition and dietetics. E-mail: epn579@uowmail.edu.au. a number of Australian dietary studies with a public health focus [7-12], however it does not include data on individual fatty acids, including LC n-3 PUFA. Currently, researchers using AUSNUT1999 who wish to also calculate LC n-3 PUFA intake utilise a fatty acid database designed to compliment AUSNUT1999 data [13]. However, the range of foods included in this database is limited, necessitating inappropriate food substitutions in studies where a wide variety of different foods are reported. Furthermore, this database does not capture the wide range of fortified food products now available to the consumer.

Whilst alternate methods exist for the calculation of LC n-3 PUFA intake, these are unsuitable for use in the Australian dietary trial context. The United States Department of Agriculture (USDA) National Nutrient Database [14] includes LC n-3 PUFA data, however 
geographical differences in the fatty acid levels of meats mean that this database is not suitable for analysing Australian data [15]. European databases inclusive of LC n-3 PUFA data similarly pose such challenges as a result of regional differences affecting fish diet and aquaculture systems [16]. Furthermore, the national food composition database, NUTrient Data Table for Use in Australia (NUTTAB95) [17], does not contain data on individual fatty acids. Whilst more recent releases of the NUTTAB databases provide data on individual fatty acids, they contain only generic food data, with no food composition data on branded food items resulting in difficulties in coding of data [18]. Conversely, as a survey database, AUSNUT1999 contains all data included in NUTTAB95, with the inclusion of a range of branded food items and additional food products reported by NNS participants, making it more appropriate for use in the dietary trial context.

AUSNUT2007 [19], a recently released database was developed to calculate nutrient intake in the National Children's Nutrition and Physical Activity Survey and included data on total LC n-3 PUFA, providing an alternate and more accurate method of calculating fatty acid intake. Measurement of the LC n-3 PUFA content of dietary intake data originally analysed using AUSNUT1999 can be achieved through the conversion of data to AUSNUT2007 [18]. To ensure accuracy and reliability, a repeatable conversion process based on a systematic approach was required.

In this study, data from a clinical trial [20] (ACTRN12608000425392, www.anzctr.org.au) was used to present a case study highlighting the importance of this database conversion. The 12 month randomised controlled trial aimed to examine the effect of increased LC n-3 PUFA intake on weight management, via supplements and dietary strategies such as increased fish and LC n-3 PUFA fortified foods. Due to database availability, all dietary intake data collected was initially analysed using AUSNUT1999, thus the database conversion was required to measure LC n-3 PUFA consumption using the AUSNUT2007. The aim of this study was to develop a defined strategy to convert dietary data from a 12 month clinical trial originally referencing the AUSNUT1999 to AUSNUT2007 and compare macronutrient intake as measured by each database. A secondary aim was to utilise the converted dietary data to estimate LC n-3 PUFA consumption in the trial.

\section{Materials and Methods}

\subsection{Study Design}

The 12 month randomised controlled trial was conducted between 2009-2010 by the BLINDED FOR REVIEW, a major coastal city 70 kilometres south of Sydney, Australia (ACTRN12608000425392, www.anzctr.org.au). Participants were randomly assigned to one of three groups: control, high dietary PUFA ("Fish”), and high dietary PUFA plus a daily LC n-3 PUFA supplement (“Fish+S”). All participants were placed on a $2 \mathrm{MJ}$ deficit daily diet and were instructed to avoid high fat foods and choose low fat staple foods. Participants in both the "Fish" and "Fish+S" groups were instructed to consume 180 grams of fish per week, with an emphasis on consumption of fatty fish high in LC n-3 PUFA and were instructed to increase their LC n-3 PUFA intake via the consumption of LC n-3 PUFA fortified foods such as bread, eggs and dairy products. The inclusion criteria for this trial were: aged 18-60 years, BMI $>25$ and $<37 \mathrm{~kg} / \mathrm{m}^{2}$, waist circumference $>94 \mathrm{~cm}$ for men, $>80 \mathrm{~cm}$ for women, generally well.

Ethical approval for the trial was granted by the University of Wollongong Human Research Ethics Committee. Written informed consent was provided by all participants.

\subsection{Database Conversion and Measurement of LC n-3 PUFA Intake}

Dietary intake information was collected by trained dietitians at baseline, three months and 12 months via a validated diet history [21]. Dietary data was initially 
entered into Foodworks (Version 5, Xyris Software, Highgate Hill, QLD, 2007), using the "AUSNUT1999 All Foods” and “AUSbrands 1999” databases [6]. The “AUSbrands 1999” database accompanies AUSNUT1999 and is inclusive of options for brand name food items, which have been matched to nutrient data from a corresponding generic food item. At the time of trial commencement, AUSNUT1999 was the most up-to-date survey specific food composition database available for use.

Conversion of nutrient data from AUSNUT1999 to AUSNUT2007 was undertaken systematically using a number of approaches (Fig. 1). Initially, all direct food item matches were made using a matching file supplied by FSANZ. Food items which were listed as "Not Further Specified" (NFS) in the 1999 database were then matched to those listed as "Not Specified" (NS) in the 2007 version.

Brand food items were matched using an additional matching file provided by FSANZ. Where exact brand names were present in the file, AUSNUT1999 foods were matched to their AUSNUT2007 counterparts. For food items where the specific brand name could not be found, or where some confusion existed, but was unlikely to alter the outcome (for example Bakers Delight Country Grain Block rather than Bakers Delight Country Grain Loaf), professional judgement was used to locate the closest substitution from the AUSNUT2007 matching file. These matches were then confirmed with the study team which consisted of five Accredited Practising Dietitians. Finally, for food items where no similar brands were available in the brand matching file (for example branded cheese and chocolate items), generic food items which were deemed to be closest from a conceptual and nutritional perspective were used via the AUSNUT2007 matching file and confirmed with the team.

Individual food items were then matched to suitable AUSNUT2007 alternatives based on conceptual and nutritional similarities (for example "Beef Mince Stew in Tom-Base Sauce from Basic Ingredients" was matched to "Beef, bolognaise pasta sauce, mince, tomato \& olive oil, homemade"). Conceptual similarities were prioritised to ensure the same form of food was chosen. Where an appropriate match for a food from the AUSNUT1999 database could not be found, a "composite food" was created. Each composite food was made up of a number of constituent

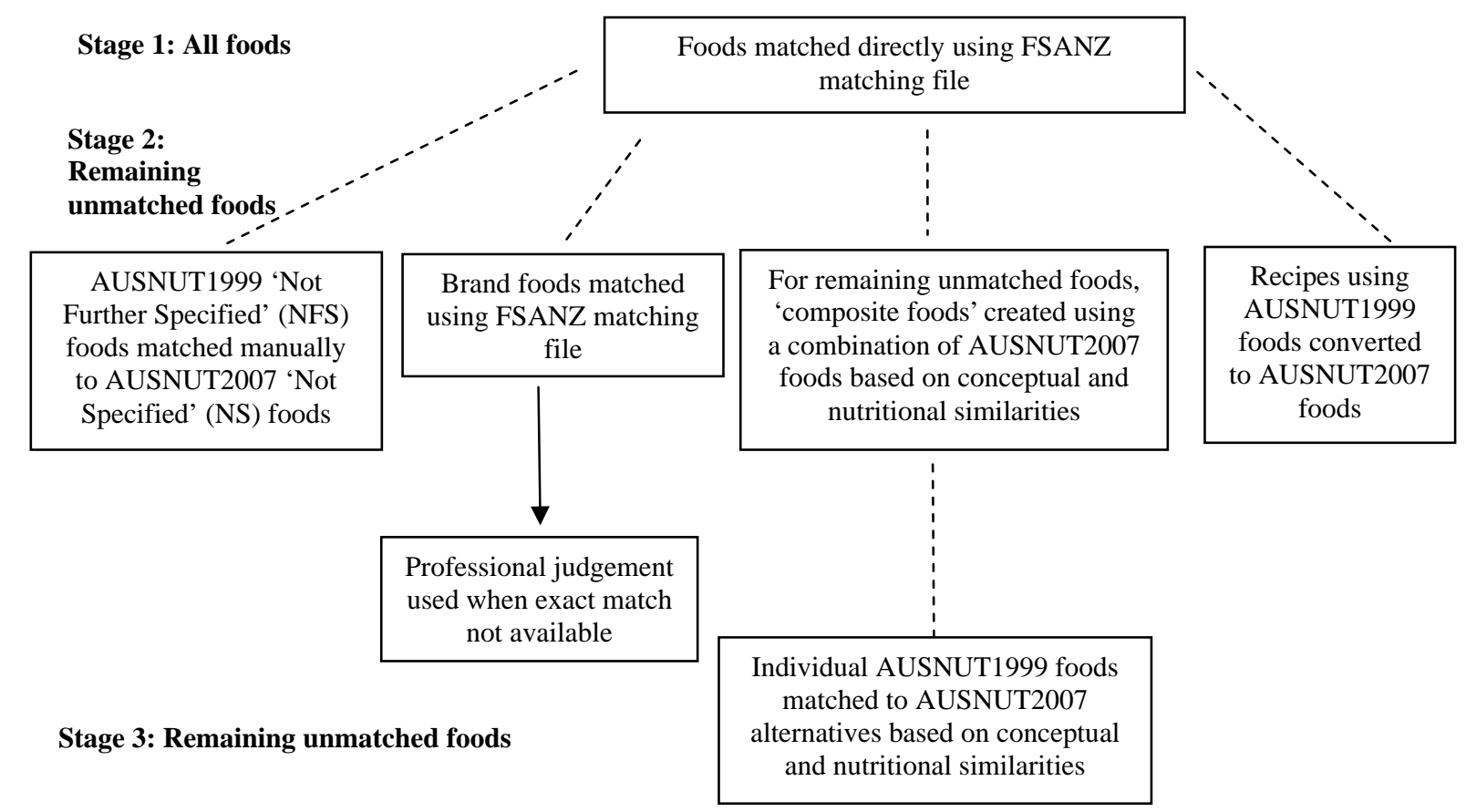

Fig. 1 The process of conversion of AUSNUT1999 dietary data to AUSNUT2007. 
AUSNUT2007 food items, which were chosen to reflect the range of potential foods or cooking methods that could be included in a single AUSNUT1999 food item (for example, a composite food designed to match the AUSNUT1999 food item "Beef, Steak, NS Cut, Grilled/Bbq, Lean \& Fat" would include a range of different beef cuts, including sirloin and rump as those most commonly consumed). Choice of the cuts of meat products used was guided by advice from relevant industry bodies. As no recent consumption data was available at the time of the present study, all foods included were weighted evenly. Care was taken to ensure that the composite food and its constituent AUSNUT2007 food items matched the original AUSNUT1999 food from both a conceptual and nutrient perspective.

Finally, recipes created using the AUSNUT1999 database were converted to consist of AUSNUT2007 foods. This was a standardised procedure which involved matching individual ingredients and portion sizes. The converted recipes were then checked to ensure their nutrient profile was similar to the original.

\subsection{Statistical Analysis}

Data was analysed using SPSS (version 17.0, SPSS Chicago, IL, 2008). Normality of the data wasidetermined using the Shapiro-Wilks test. Mean and standard deviation of energy and macronutrients including: energy, protein, total fat, saturated fat, monounsaturated fat, polyunsaturated fat, carbohydrate and fibre were calculated for both the original and converted diet histories at all time points to examine the comparability of the converted database. The difference between the original and converted diet histories was then measured using paired samples $t$-tests and Wilcoxon signed ranks test.

The change in LC n-3 PUFA intake over the duration of the trial was determined using the Friedman test. Where significant differences were noted, post-hoc tests with Bonferroni adjustments were run to investigate the source of the variation.

\section{Results and Discussion}

\subsection{Results}

Data on diet histories was available for this analysis from $\mathrm{n}=118$ participants at baseline, $\mathrm{n}=86$ at three months, and $n=64$ at 12 months. Missing data was the result of study dropouts, however as this was a secondary analysis of a randomised controlled trial, data analysis was performed on the subset of data available. Foods were matched using a number of approaches at each time point (Table 1).

At baseline, all macronutrients measured in the AUSNUT2007 converted diet histories were significantly different to the original AUSNUT1999 diet histories (Table 2). At three months, fat subtypes in the converted diet histories were not significantly different from the original diet histories, whilst the other macronutrients measured were significantly different between the original and converted diet histories. By twelve months, protein and fibre were the only macronutrients that were significantly different between the original and converted diet histories.

Mean \pm SD LC n-3 PUFA intake was $441.87 \mathrm{mg} \pm$ 424.81 at baseline, $521.07 \mathrm{mg} \pm 492.36$ at three months, and $442.40 \mathrm{mg} \pm 300.14$ at 12 months. Friedman test indicated a change in LC n-3 PUFA intake over the duration of the study ( $P=0.047$ ), but after performing post-hoc tests with Bonferroni adjustment this change was no longer significant.

\subsection{Discussion}

This is the first known study to convert Australian dietary intake data from AUSNUT1999 to 2007 via a systematic approach and illustrate its practical use. This process allowed for the measurement of the LC n-3 PUFA content of the diet, which was previously impossible in earlier versions of Australian nutrient databases. Given the wide-spread use of AUSNUT1999 in public health literature [7-12], this conversion process could be of use to many Australian researchers to measure fatty acid intake in previously 
Table 1 Food items (n) matched by the systematic approach at baseline, three and twelve months.

\begin{tabular}{|c|c|c|c|}
\hline & Baseline & Three months & Twelve months \\
\hline Total number of foods requiring conversion (individual food items) & $8232(1614)$ & $5258(1094)$ & $3478(735)$ \\
\hline $\begin{array}{l}\text { Foods matched using matching file developed by FSANZ } \\
\text { (for example "Salmon, baked or grilled, fat not added in cooking” [1999] } \\
\text { matched to "Salmon, Atlantic, fillet, grilled" [2007]) }\end{array}$ & 635 & 454 & 397 \\
\hline $\begin{array}{l}\text { Brand foods matched using matching file } \\
\text { (for example "Arnotts butternut cookie" [1999] matched to "Biscuit, sweet, } \\
\text { Anzac or butternut style” [2007]) }\end{array}$ & 217 & 119 & 109 \\
\hline $\begin{array}{l}\text { Foods matched using professional judgement } \\
\text { (for example "Dim sim, meat-filled, deep fried" [1999] matched to "Dim sim, } \\
\text { meat and vegetable filling, deep fried” [2007]) }\end{array}$ & 577 & 385 & 338 \\
\hline $\begin{array}{l}\text { Foods requiring development of a new composite foods } \\
\text { (for example "Meat curry, NFS" [1999] matched to a new composite food } \\
\text { consisting of "Beef, curry, prepared with curry powder, onions \& stock" } \\
\text { [2007], “Chicken, curry, butter, Indian restaurant style" [2007], "Lamb, } \\
\text { curry, korma, Indian restaurant style” [2007]) }\end{array}$ & 135 & 88 & 83 \\
\hline $\begin{array}{l}\text { AUSNUT1999 recipes requiring conversion } \\
\text { (for example, constituent ingredients of "Sushi roll, crab, avocado" [1999] } \\
\text { matched to "Seaweed, nori, dried" [2007], "Rice, white, boiled without added } \\
\text { salt" [2007], "Crab, various types, fresh only, boiled or steamed" [2007], } \\
\text { "Avocado, raw, not further specified" [2007] based on original portion sizes) }\end{array}$ & 132 & 48 & 36 \\
\hline
\end{tabular}

Table 2 Mean \pm standard deviation daily energy and macronutrient intake estimated by the original and converted diet histories at baseline, three and twelve months.

\begin{tabular}{|c|c|c|c|c|c|c|c|c|c|}
\hline & \multicolumn{2}{|c|}{ Baseline } & \multirow{2}{*}{-P-value } & \multicolumn{2}{|c|}{3 months } & \multirow{2}{*}{-P-value } & \multicolumn{2}{|c|}{12 months } & \multirow{2}{*}{-P-value } \\
\hline & Original & Converted & & Original & Converted & & Original & Converted & \\
\hline $\begin{array}{l}\text { Energy } \\
\text { (kJ/day) }\end{array}$ & $9973.05 \pm 3.45$ & $9757.73 \pm 3.30$ & $0.000^{* *}$ & $6533.98 \pm 1.43$ & $6479.10 \pm 1.42$ & $0.018^{* *}$ & $6901.86 \pm 1.66$ & $6889.00 \pm 1.70$ & $0.682^{*}$ \\
\hline Protein (g/day) & $106.95 \pm 37.70$ & $110.51 \pm 39.27$ & $0.000^{* *}$ & $86.06 \pm 22.05$ & $89.18 \pm 22.63$ & $0.000^{* *}$ & $87.11 \pm 22.06$ & $90.56 \pm 23.80$ & $0.000^{*}$ \\
\hline $\begin{array}{l}\text { Total fat } \\
\text { (g/day) }\end{array}$ & $93.89 \pm 42.01$ & $88.76 \pm 39.03$ & $0.000^{* *}$ & $45.77 \pm 15.64$ & $44.26 \pm 15.40$ & $0.000^{* *}$ & $50.65 \pm 18.36$ & $49.34 \pm 18.15$ & $0.071^{* *}$ \\
\hline $\begin{array}{l}\text { Saturated fat } \\
\text { (g/day) }\end{array}$ & $35.05 \pm 19.16$ & $33.00 \pm 17.44$ & $0.000^{* *}$ & $14.07 \pm 4.88$ & $13.68 \pm 4.89$ & $0.113^{* *}$ & $16.06 \pm 6.76$ & $15.83 \pm 6.35$ & $0.525^{* *}$ \\
\hline PUFA (g/day) & $14.88 \pm 8.00$ & $14.45 \pm 8.29$ & $0.042^{* *}$ & $9.83 \pm 5.66$ & $9.92 \pm 5.46$ & $0.463^{*}$ & $10.26 \pm 7.36$ & $10.25 \pm 6.82$ & $0.664^{* *}$ \\
\hline MUFA (g/day) & $35.85 \pm 15.97$ & $34.85 \pm 14.98$ & $0.001^{* *}$ & $17.11 \pm 6.96$ & $16.85 \pm 6.92$ & $0.118^{* *}$ & $19.15 \pm 7.62$ & $19.22 \pm 7.89$ & $0.915^{* *}$ \\
\hline CHO (g/day) & $243.53 \pm 82.73$ & $241.61 \pm 81.97$ & $0.009^{* *}$ & $174.32 \pm 40.54$ & $171.88 \pm 40.28$ & $0.001^{* *}$ & $181.47 \pm 45.18$ & $180.69 \pm 47.23$ & $0.376^{*}$ \\
\hline Fibre (g/day) & $27.60 \pm 10.25$ & $29.74 \pm 11.32$ & $0.000^{* *}$ & $25.19 \pm 5.65$ & $27.98 \pm 6.49$ & $0.000^{*}$ & $25.21 \pm 5.94$ & $28.68 \pm 7.69$ & $0.000^{*}$ \\
\hline
\end{tabular}

*: paired samples $t$-test.

**: Wilcoxon signed ranks test.

collected data. Furthermore, the methodologies developed in this study could also be adapted to be applicable to other nutrients now available in updated food composition databases.

The database conversion undertaken in this study provides an alternative to previous strategies of measuring the LC n-3 PUFA content of the diet, such as the fatty acid database developed by Mann et al. [13]. Whilst this database could also be used to calculate the LC n-3 PUFA content of dietary data originally analysed using AUSNUT1999, the limited range of foods included means it is not viable as a method for measuring LC n-3 PUFA intake from open-ended diet histories, which typically contain a wide variety of different foods [22]. The current database conversion overcomes this problem by matching all foods systematically.

The accuracy of the conversion process appeared to be highest at the 12 month time point of the clinical trial. Whilst several significant differences were noted between the original and converted diet histories at baseline, there were no significant differences in the values for energy, saturated fat, polyunsaturated fat, monounsaturated fat and carbohydrate at twelve months (Table 2). This is likely to be the result of increased dietitian experience during the progression 
of the clinical trial. Analysis of diet histories entered at baseline suggests that generic food options were often used (for example, "meat, cooked, nfs"), introducing more room for error whilst matching these foods to AUSNUT2007 alternatives. In comparison, at the twelve month time point, more specific food items were used, thus making it more likely that a suitable alternative was chosen via professional judgement for the database conversion.

The results of the database conversion also provide insight into the implementation of the conversion process. Fat subtypes appeared to be the closest matched macronutrients, with no significant differences noted between the original and converted databases at three and twelve months (Table 2). This reflects the purpose of the conversion process, as the current clinical trial focused on fat consumption, meaning that emphasis was placed on ensuring that matches made using professional judgement were closest in fat sub-categories. Conversely macronutrients such as protein and fibre were significantly different at all time points, suggesting that the current approach may not be optimal for researchers attempting to measure these nutrients as a primary outcome.

The practical implementation of the database conversion process was highlighted in the measurement of LC n-3 PUFA consumption in this study. Mean LC n-3 PUFA intake was highest at the three month time point; although a wide range in LC n-3 PUFA consumption was seen. The mean intake of LC n-3 PUFA at all time points in the present study was higher than that measured in a representative national survey conducted in 1995 [15]. Using the previously discussed fatty acid database, Howe et al. [15] analysed the results of the 1995 National Nutrition Survey (NNS) and found a mean consumption of $246 \pm$ SEM $5 \mathrm{mg} /$ day for adults. This difference may be due to the current study participants being volunteers for a dietetic intervention, thus their eating habits may not be indicative of the general population. Furthermore, the differences in study results may be due to changes in eating habits in the 15 years between the NNS and the current study. Given that recommendations for increased fish consumption have been released during this time period [23], eating habits may have changed to reflect this. However as there have been no population wide Australian dietary surveys since the NNS, this cannot be confirmed.

Whilst the database conversion outlined in this study was based on a systematic approach, many of the choices were based on subjective professional judgement, which could be considered a limitation of this research. However, all decisions were confirmed with the study team of research dietitians before being finalised, which decreases the risk of bias. An additional limitation is the variety of foods available in the AUSNUT2007 database, which was developed to calculate nutrient intake from the 'Kids Eat Kids Play' survey of Australian children. As a result of this, categories of some foods not regularly consumed by children, for example alcohol, are limited, and thus finding an appropriate match for such AUSNUT1999 foods is problematic.

\section{Conclusion}

Conversion of dietary trial data from AUSNUT1999 to AUSNUT2007 databases via a systematic approach was found to result in comparable macronutrient values, particularly at the 12 month time point. At the 12 months, there were no significant differences between the original and converted databases in measures of total energy, total fat, saturated fat, PUFA, MUFA and carbohydrate. Implementation of this database conversion allowed for the measurement of LC n-3 PUFA over the duration of a dietary trial and in a wider range of food items than previously available databases. Mean \pm SD LC n-3 PUFA intake was $441.87 \mathrm{mg} \pm 424.81$ at baseline, $521.07 \mathrm{mg} \pm 492.36$ at three months, and $442.40 \mathrm{mg} \pm 300.14$ at 12 months. This methodology 
could be adapted in other Australian studies to allow for the measurement of LC n-3 PUFA intake or other nutrients in previously collected data.

\section{Acknowledgments}

The authors declare they have no competing interests. This work was supported by the National Health and Medical Research Council (514631). Elizabeth Neale was supported by an Australian Post-graduate Award.

\section{References}

[1] D. Mozaffarian, A. Ascherio, F.B. Hu, M.J. Stampfer, W.C. Willett, D.S. Siscovick, et al., Interplay between different polyunsaturated fatty acids and risk of coronary heart disease in men, Circulation 111 (2005) 157-164.

[2] F.B. Hu, L. Bronner, W.C. Willett, M.J. Stampfer, K.M. Rexrode, C.M. Albert, et al., Fish and Omega-3 fatty acid intake and risk of coronary heart disease in women, JAMA 287 (2002) 1815-1821.

[3] H. Iso, K.M. Rexrode, M.J. Stampfer, J.E. Manson, G.A. Colditz, F.E. Speizer, et al., Intake of fish and omega-3 fatty acids and risk of stroke in women, JAMA 285 (2001) 304-312.

[4] E.B. Levitan, A. Wolk, M.A. Mittleman, Fish consumption, marine omega-3 fatty acids, and incidence of heart failure: A population-based prospective study of middle-aged and elderly men, Eur. Heart J. 30 (2009) 1495-1500.

[5] K. Yamagishi, H. Iso, C. Date, M. Fukui, K. Wakai, S. Kikuchi, et al., Fish, [omega]-3 polyunsaturated fatty acids, and mortality from cardiovascular diseases in a nationwide community-based cohort of Japanese men and women: The JACC (Japan Collaborative Cohort Study for Evaluation of Cancer Risk) study, Journal of the American College of Cardiology 52 (2008) 988-996.

[6] Food Standards Australia and New Zealand, AUSNUT1999-Australian Food and Nutrient Database 1999 for Estimation of Dietary Intake , Canberra, 1999.

[7] K. Charlton, H. Yeatman, F. Houweling, S. Guenon, Urinary sodium excretion, dietary sources of sodium intake and knowledge and practices around salt use in a group of healthy Australian women, Australian and New Zealand Journal of Public Health 34 (2010) 356-363.

[8] J.K. Brimblecombe, The role of energy cost in food choices for an Aboriginal population in Northern Australia, Medical Journal of Australia 190 (2009) 549.

[9] C. Bower, M. Miller, J. Payne, P. Serna, N. de Klerk, F.J. Stanley, Folate promotion in Western Australia and the prevention of neural tube defects, Australian and New Zealand Journal of Public Health 28 (2004) 458-464.
[10] P. Morgan, C. Collins, R. Plotnikoff, P. McElduff, T. Burrows, J. Warren, et al., The SHED-IT community trial study protocol: A randomised controlled trial of weight loss programs for overweight and obese men, BMC Public Health 10 (2010) 701.

[11] S. Pal, C. Cheng, S. Ho, The effect of two different health messages on physical activity levels and health in sedentary overweight, middle-aged women, BMC Public Health 11 (2011) 204.

[12] L. Hall, C.E. Collins, P.J. Morgan, T.L. Burrows, D.R. Lubans, R. Callister, Children's intake of fruit and selected energy-dense nutrient-poor foods is associated with fathers' intake, Journal of the American Dietetic Association 111 (2011) 1039-1044.

[13] N. Mann, A. Sinclair, P. Percival, J. Lewis, B. Meyer, P. Howe, Development of a database of fatty acids in Australian foods, Nutrition and Dietetics 60 (2003) 42-45.

[14] U.S. Department of Agriculture, Agriculture Research Service, 2005.

[15] P. Howe, B. Meyer, S. Record, K. Baghurst, Dietary intake of long-chain [omega]-3 polyunsaturated fatty acids: contribution of meat sources, Nutrition 22 (2006) 47-53.

[16] P.M. Kris-Etherton, W.S. Harris, L.J. Appel, C. for the nutrition, fish consumption, fish oil, omega-3 fatty acids and Cardiovascular disease, Circulation 106 (2002) 2747-2757.

[17] Food Standards Australia and New Zealand, NUTTAB95-NUTrient Data Table for Use in Australia, Canberra, 1995.

[18] R. Sobolewski, J. Cunningham, D. Mackerras, Which Australian food composition database should I use?, Nutrition \& Dietetics 67 (2010) 37-40.

[19] Food Standards Australia and New Zealand, AUSNUT 2007-Australian Food, Supplement and Nutrient Database for Estimation of Population Nutrient Intakes, Canberra, 2008.

[20] L.C. Tapsell, M.J. Batterham, K.E. Charlton, Effects of n-3 fatty acids on weight loss: A 1 year randomized controlled trial, The FASEB Journal 25 (2011) 774-775.

[21] G.S. Martin, L.C. Tapsell, S. Denmeade, M.J. Batterham, Relative validity of a diet history interview in an intervention trial manipulating dietary fat in the management of Type II diabetes mellitus [small star, filled], Preventive Medicine 36 (2003) 420-428.

[22] A.E. Black, A.A. Welch, S.A. Bingham, Validation of dietary intakes measured by diet history against $24 \mathrm{~h}$ urinary nitrogen excretion and energy expenditure measuredby the doubly-labelled water method in middle-aged women, British Journal of Nutrition 83 (2000) 341-354.

[23] National Heart Foundation of Australia, A review of the relationship between dietary fat and cardiovascular disease, Australian Journal of Nutrition and Dietetics 56 (1999) S5-S22. 